\title{
No difference between full-mouth and quadrant-wise treatment of chronic periodontitis
}

\author{
Abstracted from \\ Farman M, Joshi RI. \\ Full-mouth treatment versus quadrant root surface debridement in the treatment of chronic periodontitis: \\ a systematic review. Br Dent / 2008; 9: 205:496-497(on line article E18) \\ Address for correspondence: Mr. Rajendra Joshi, Consultant in Restorative Dentistry, Charles Clifford Dental Hospital, \\ Wellesley Road, Sheffield S10 2SZ, UK. E-mail: Raj.Joshi@sth.nhs.uk
}

\section{Question: In the treatment of chronic periodontitis, is full-mouth debridement and/ or disinfection more effective than quadrant scaling and root planing?}

\section{Data Sources Medline and Embase, along with reference lists from retrieved papers, were used to source relevant studies.}

Study selection Only English-language randomised controlled clinical trials (RCT) with at least 6 months followup; that used the patient as the unit of analysis; included patients with chronic periodontitis who had not used antibiotics for 3 months prior to the start of the study; and compared fullmouth debridement or full-mouth disinfection with conventional quadrant root planing were included.

Outcome measure Measures considered were reductions in probing pocket depths, probing attachment levels and bleeding on probing. Study quality was assessed based on randomisation methods, allocation concealment, examiner blinding and completeness of followup. Data extraction and synthesis Data were extracted by two reviewers independently and meta-analysis was performed.

Results Seven RCT were included. There were no statistically significant differences between the full-mouth debridement and quadrant approaches. Conclusions The review suggests that mechanical or nonsurgical periodontal treatment is effective but showed no difference in the periodontal clinical outcome measures between the full-mouth debridement and quadrant approaches.

\section{Commentary}

High-quality systematic reviews provide a more objective and complete view of the research literature than that provided by a standard "narrative" review and facilitate the transfer of research knowledge to clinical practice. This review by Farman and Joshi is carried out well, in following (for the most part) the recommendations set out in the QUOROM statement. ${ }^{1}$ Its major flaw is with the search. As Glenny and colleagues pointed out, ${ }^{2}$ systematic reviews should be designed to identify all published and unpublished data, irrespective of language. This is an admirable aim, though not always feasible because of restriction of resources, because exclusion of non-English databases can introduce publication bias. Furthermore, a combination of a Medline search and searching journals by hand has been shown to identify nearly twice as many clinical trials as database searching alone. ${ }^{3}$ Even though Farman and Joshi did manually search references lists of retrieved papers, they did not search periodontal journals, which could further contribute to publication bias.
Periodontists are constantly trying to discover more exciting, effective or efficient ways to clean the "schmutz" from diseased root surfaces. The aim of this review is to determine whether adding oral antiseptics to root planing, and/ or completing the debridement in fewer appointments, is as efficacious as the traditional quadrantbased protocol. The meta-analysis revealed that, although adding oral antiseptics such as chlorhexidine or iodine might be slightly more exciting, it is certainly no more effective. And although fullmouth debridement may provide some cost saving in terms of time for the clinician and patient, the risk of increased pain from this procedure may outweigh the benefit.

As with so many systematic reviews, the diversity of experimental design and treatment protocols limited the inclusion of a number of studies. Further, among those that qualified for inclusion in the review, only two out of the seven met the quality standards for methodology. Thus, the lack of any statistically significant advantage of one technique over another could well be a result of poor study design. The CONSORT statement ${ }^{4}$ outlining guidelines for carrying out clinical trials was published in 2001. Despite this, trials without adequate allocation concealment or randomisation, sample size calculations or intent-to-treat analysis are still being conducted and published. Data from these types of studies dilute the potential power of a systematic review in clinical decision making. It frustrates the reviewers and confuses the average practitioner. As a community, we need to continue the push for high-quality evidence through education of future (and current) researchers and appropriate standards for publication.

\section{Debora C Matthews}

Division of Periodontics, Dalhousie University, Halifax, Canada

\section{Practice points}

- The addition of oral antiseptics to mechanical debridement offers no advantage over scaling and root planing alone.

- Full-mouth debridement may take less time to complete than quadrant scaling and root planing, but may also increase patients' post-operative pain.

1. Moher D, Cook Dl, Eastwood S, Olkin I, Rennie D, Stroup DF. Improving the quality of reports of meta-analyses of randomised controlled trials: the QUOROM statement. Quality of Reporting of Meta-analyses. Lancet 1999; 354: 1896-1900.

2. Glenny AM, Esposito M, Coulthard P, Worthington HV. The assessment of systematic reviews in dentistry. Eur / Oral Sciences 2003; 111: 85-92.

3. Bickley SR, Harrison JE. How to... find the evidence. J Orthod 2003; 30: 72-78.

4. Moher D, Schulz KF, Altman DG. The CONSORT statement: revised recommendations for improving the quality of reports of parallel-group randomised trials. Lancet 2001; 357(9263): 1191-1194.

Evidence-Based Dentistry (2009) 10, 17. doi:10.1038/sj.ebd.6400631 